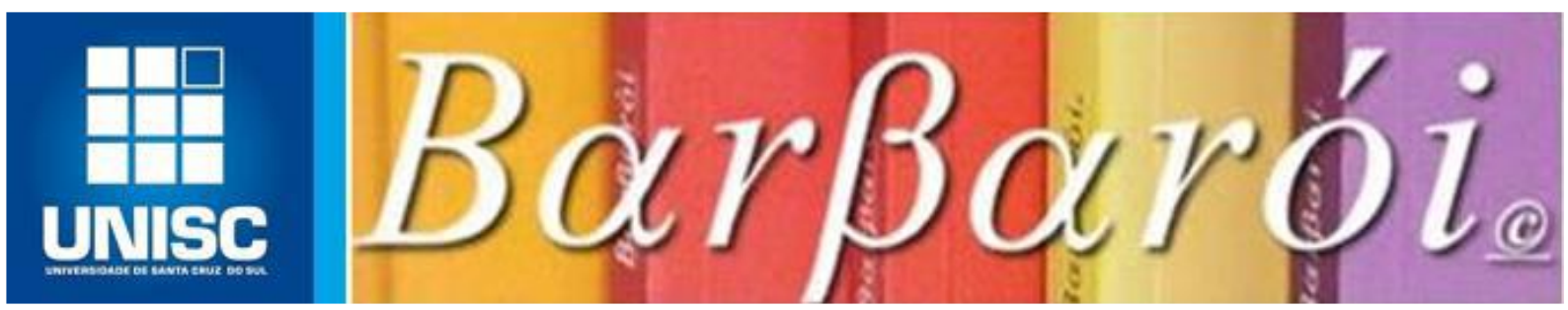

\title{
CONTRIBUIÇÕES DA EDUCAÇÃO PROFISSIONAL NA ENFERMAGEM PARA O ENFRENTAMENTO DA ESTIGMATIZAÇÃO ASSOCIADA AOS TRANSTORNOS MENTAIS
}

\section{DOI: http://dx.doi.org/10.17058/barbaroi.v0i0.13281}

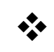 \\ Marcela Santos Ferreira \\ Centro Federal de Educação Tecnológica Celso Suckow da Fonseca - CEFET/RJ-Brasil \\ Maria Cecilia Araujo Carvalho \\ Escola Politécnica de Saúde Joaquim Venâncio - EPSJV/FIOCRUZ - Brasil

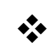

\section{Resumo}

As atitudes estigmatizantes associadas aos portadores de transtornos mentais presentes em estudantes e trabalhadores da área da saúde são reproduzidas no cuidado a saúde, produzindo sofrimento, além daquele gerado pelo próprio transtorno mental. Uma forma de enfrentamento desse problema social é o investimento da educação profissional em um ensino antiestigmatizante. Para contemplar a temática foi realizada uma revisão de literatura de forma que os seguintes objetivos da pesquisa fossem alcançados, que é conceber apontamentos teóricos e práticos sobre como a educação, durante a formação profissional, pode ser uma ferramenta de enfrentamento do estigma relacionado aos portadores de transtornos mentais, presente na área da enfermagem. A argumentação central do artigo é que a formação profissional é um momento importante para o combate da estigmatização aos portadores de transtornos mentais. Para tanto, é imprescindível que o ensino de saúde mental seja baseado em uma educação crítica, tenha seus conteúdos formativos adequados a um contexto antiestigmatizante e associe várias estratégias educacionais voltadas para a redução do estigma, como ocorre na estratégia de contato com portadores de transtornos mentais. A educação profissional na enfermagem quando em consonância com uma prática antiestigmatizante é capaz de restringir a ação do estigma associado ao transtorno mental, por parte dos profissionais da Enfermagem. A consequência desse tipo de formação é a melhora da assistência devido a um cuidado em saúde com menos estigma.

Palavras-chave: Estigma social; Transtorno mental; Educação; Enfermagem. 


\section{Introdução}

O estigma é um processo social que comumente está associado aos transtornos mentais, sendo responsável por uma queda na qualidade de vida das pessoas acometidas por esses problemas. As atitudes estigmatizantes são encontradas na população em geral, não excluindo estudantes e profissionais da saúde que ao reproduzirem na assistência essa estigmatização, torna os indivíduos, com algum sofrimento psíquico, mais vulneráveis a não ter um cuidado integral e de excelência.

O estigma conforme Ahmedani (2011, p. 2), "É entendido como opiniões negativas atribuídas a uma pessoa ou grupos de pessoas ou quando as suas características comportamentais são vistas como diferentes as normas sociais”. Essa forma de representação sobre os portadores de transtornos mentais é encontrada em atitudes de alguns trabalhadores e estudantes da área da saúde.

De acordo com Batista (2013, p. 14), "O pessimismo relativamente ao tratamento e prognóstico e o desejo de distância social, em particular, parecem ser as atitudes estigmatizantes mais prevalentes entre os profissionais de saúde". Já os estudos de Ahmedani (2011), evidencia que os profissionais de saúde podem apresentar o mesmo estigma encontrado pela população em geral, e que o desejo de manter uma distância dos portadores de transtornos mentais não diferiu, ao comparar com o comportamento do resto da população. E segundo o autor, os usuários dos serviços de saúde ao serem marginalizados pelo processo de estigmatização tornam-se mais propensos a acabar o tratamento atual e menos dispostos a procurar tratamento no futuro.

Apesar de serem profissionais da saúde, antes de tudo são pessoas, que desde a infância desenvolvem crenças sobre si elas, sobre os outros e sobre o mundo (GIL, 2010). Essas crenças, consideradas informações que estão entre o conhecimento popular e científico, relacionam-se ao estigma durante a vida profissional, pois um dos problemas associados ao estigma é o conhecimento, segundo Thornicroft et al. (2007).

Em se tratando de conhecimento, a educação profissional tem uma grande responsabilidade na perpetuação ou transformação de conhecimentos estereotipados sobre os portadores de transtornos mentais, o que gera a permanência ou não das crenças anteriores à formação e o medo do desconhecido. Segundo Lucchese e Barros (2009), os profissionais podem não estar preparados para prestar assistência a este tipo de clientela devido à falta de especialização, treinamento ou até mesmo a falta de conhecimento proporcionada pela baixa carga horária dispensada pelas escolas para o ensino da saúde mental. 
Faz-se necessário a melhoria da formação profissional não só voltada para os profissionais da Enfermagem especialistas em saúde mental e sim para todos os profissionais de saúde e outros que estejam comprometidos com assistência a essa população. Pois se sabe que parte da população com transtorno mental, antes de ser atendida em unidades especializadas, passam pela atenção básica.

A presente investigação visa a buscar, por meio de busca na literatura, conteúdos teóricos que possam basear uma educação voltada para a estigmatização, assim como, práticas já estabelecidas no campo da educação em saúde.

Desta forma, o objetivo deste estudo foi conceber apontamentos teóricos e práticos sobre como a educação, durante a formação profissional, pode ser uma ferramenta de enfrentamento do estigma relacionado aos portadores de transtornos mentais, presente na área da enfermagem.

\section{Método}

A revisão de literatura é a busca de informações sobre um determinado tema, com a finalidade de permitir a tradução das melhores evidências científicas nas políticas, práticas e decisões no contexto da saúde e proporcionar ao leitor uma compreensão do que existe publicado sobre o assunto (POLIT; HUNGLER, 2004).

Para atender os objetivos deste estudo, realizou-se uma revisão da literatura, com base nos seguintes passos metodológicos: 1. seleção da questão para a revisão; 2 . seleção da amostra; 3. definição das características dos estudos da amostra com base em critérios de inclusão definidos pelos autores; 4. Análise e discussão dos resultados.

Os critérios de inclusão para definição da amostra deste estudo foram: trabalhos publicados na integra com temática voltada para contribuição da educação na Enfermagem para combate ao estigma relacionado ao portador de transtorno mental, em periódicos nacionais e internacionais; estudos empíricos e teóricos.

As publicações que compuseram a amostra foram encontradas nas bases de dados relacionadas Lilacs, Scopus, Medline, Pubmed e Scielo, sendo utilizado para a busca o termo estigma e outros que foram cruzados a ele, utilizando o operador booleano AND com a finalidade de restringir a pesquisa aos resumos que apresentavam ao mesmo tempo os termos estigma e transtorno mental, doença mental, sofrimento psíquico, atitude, educação, formação profissional, enfermagem. Foi utilizado o dicionário de palavras de cada base, o que proporcionou as palavras-chaves indexadas adequadas para as buscas. $\mathrm{O}$ acesso aos 
periódicos internacionais e nacionais foi obtido através do Portal de Periódicos da Coordenação de Aperfeiçoamento de Pessoal de Nível Superior.

Um total de 874 estudos foi localizado na busca inicial nas bases de dados. Após a eliminação de estudos duplicados, realizou-se a leitura de todos os títulos e resumos, seguiram-se os critérios de inclusão e, ao final, sete estudos atenderam esses critérios e foram selecionados.

\section{Enfrentamento do estigma por uma educação profissional crítica}

A educação profissional, por meio do ensino de saúde mental, é um importante instrumento para confrontar inúmeros tipos de estigma e quando contextualizada com as propostas atuais de assistência ao portador de transtorno mental, pode oferecer ao estudante ou profissional de saúde base para diminuir seus próprios estigmas, como também ser um ativista contra o estigma social que esta população experimenta.

A educação é considerada por Arboleda-Flórez e Stuart (2012, p. 461) uma das abordagens para a redução do estigma e, que, ao ser aplicada à população, seja composta por leigos, portadores de transtornos mentais, estudantes ou profissionais de saúde, tem como propósito "substituir mitos e a desinformação com concepções precisas sobre a natureza e a prevalência de transtornos mentais, melhorando assim a carga de conhecimento global em saúde mental".

Para que o ensino possa cumprir os propósitos citados, algumas mudanças na trajetória educacional devem ocorrer com o intuito de desenvolver junto aos profissionais da saúde, formados ou ainda em formação, entendimento necessário para que os elementos abrangentes do estigma, como o conhecimento, preconceito e a discriminação possam ser limitados.

O ensino de saúde mental deve estar em consonância com as mudanças ocorridas, principalmente com a reforma psiquiátrica, no entanto, para que isso ocorra, deve haver uma reorientação do que é ensinado. Apesar de na prática assistencial existirem novos olhares sobre a pessoa com sofrimento psíquico, o saber psiquiátrico tradicional ainda domina parte da formação de profissionais de saúde. A formação deve ser pensada e ir além de práticas médicas com concepções normatizantes e biologicistas.

A reflexão sobre a construção de um ensino de saúde mental baseada nesse novo paradigma deve partir de algumas indagações cujas respostas guiam essa reforma do ensino: "Quem estamos pretendendo formar? Para que estamos pretendendo formar? Para quem estamos formando? Qual o objetivo de nossa formação? O que pretendemos mudar ou introduzir a partir de nossa formação?" (AMARANTE, 2008, p.66).

Barbarói, Santa Cruz do Sul, n. 56, p.<127-140>,jan./jun. 2020 
Todavia, existem alguns obstáculos encontrados na educação profissional, observados no dia a dia, que dificultam a formação comprometida com essas atuais realidades. O preparo do docente, apoio institucional, projeto pedagógico, referências pedagógicas, entre outros fatores que podem interferir de tal modo que o currículo objetive adaptar os estudantes e futuros técnicos em saúde para atuarem em uma psiquiatria hospitalocêntrica, medicalizadora e centrada no transtorno mental, o que na prática leva a repetir antigas atitudes estigmatizantes, provocando dificuldades na inserção desse trabalhador na atenção à Saúde Mental que propõe um cuidado psicossocial não asilar ao sujeito em sofrimento psíquico.

Da mesma forma que a psiquiatria normalizadora e biologicista domina parte do ensino de saúde mental, a pedagogia tradicional também detém esse poder de tal forma que, dificulta o desenvolvimento de práticas educativas que objetivam a reflexão dos conteúdos sobre saúde mental e estigma. A atuação dessa pedagogia no ensino dos trabalhadores da enfermagem é bem demostrada por Lucchese e Barros (2009, p. 156) quando consideram que a "pedagogia tradicional prioriza os procedimentos técnicos por meio um ensino fracionado, contrapondo-o a um processo de formação integral, essencial para a atenção psicossocial”.

Objetivar mudanças nas atitudes estigmatizantes de estudantes ou profissionais da saúde mantendo essa estrutura tradicional de ensinar saúde mental torna-se difícil, pois é a transformação que demanda, em algumas situações, o abandono de algumas crenças e para isso fazem-se necessárias práticas educativas que a pedagogia tradicional não contempla. De forma a buscar um novo caminho para esse ensino na enfermagem, mas que pode ser aplicado a qualquer profissão que atua na rede de atenção a saúde mental, Lucchese e Barros (2009, p. 156) propõem que:

É o momento de o educador em enfermagem buscar novas estratégias de ensino que proporcionem uma situação de aprendizagem, mediante ações transformadoras. É necessário desenvolver o pensamento crítico e questionador, estimular a liderança, explorar as potencialidades de ser humano em cada aluno, empregando estratégias problematizadoras [...].

Apesar das dificuldades, inúmeros estudos mostram que a formação profissional de caráter emancipador e de perspectiva crítica influencia de modo positivo a redução dos estigmas associados ao transtorno mental. Gil (2010, p. 62) mostra a partir de um estudo comparativo entre o início e o fim de um curso de graduação em enfermagem em Portugal que "as atitudes mais positivas face à doença mental são constatadas nos finalistas do referido curso, o que sugere a influência que a formação acadêmica pode ter na 'mudança' de atitudes". 


\section{Adequações do ensino de saúde mental como base para transformações das atitudes estigmatizantes}

Ao se construir um conteúdo programático voltado para o ensino de saúde mental, deve-se pensar em como a temática estigma estará inserida nesse ensino. Estudos que avaliam a influência do ensino de saúde mental nas atitudes estigmatizantes pré e pós-ensino mostram que a maioria é naturalista, ou seja, nenhuma intervenção foi adicionada ao que cada programa oferecia (GYLLENSTEN et al., 2011).

De acordo com Thornicroft et al. (2008), as intervenções educacionais com os melhores resultados são aquelas integradas ao currículo tradicional, o que remete à reflexão sobre a necessidade de incorporação da discussão do estigma no currículo como forma de determinar uma maior influência do ensino sob as atitudes de estudantes ou profissionais de saúde.

Para que uma intervenção educacional cumpra seu propósito de redução do estigma é necessário que o discente tenha contato com conhecimentos referentes aos conceitos, tipos, causas, consequências e fatores que promovem a resistência do estigma, como também se faz necessário que este seja entendido como um determinante social para a saúde mental, levando à compreensão de que parte das estratégias de reabilitação psicossocial do transtorno mental passa pelo enfrentamento do estigma.

Um ponto de partida importante para discussão de estigma é o conhecimento dos processos históricos que envolvem os portadores de transtornos mentais e a estigmatização associada a eles. A lógica de como esses processos se formou na sociedade é útil para melhorar a compreensão da situação atual do estigma. A percepção histórica da loucura por parte dos alunos leva-os ao caminho da assimilação de que o estigma tem suas origens muito antes da psiquiatria como ciência, apesar de que essa, com suas transformações, influenciou a forma como a sociedade enxerga os portadores de transtornos mentais, traduzido em uma imagem estereotipada e estigmatizadora da loucura, tratando-os como irracionais, incapazes e perigosos.

Em se tratando de processo histórico, a reforma psiquiátrica, assim como suas repercussões, é uma temática crucial no debate sobre estigma, especialmente para os estudantes mais jovens. De acordo com Bezerra Junior (2007) O ensino dos jovens pode ser um desafio fundamental para a formação de recursos humanos para esta área, pois os mesmos não passaram pelo processo de luta política e ideológica que envolveu a criação do movimento antimanicomial 
[...] Boa parte desses profissionais se tornou adulta num momento da vida do país em que as grandes bandeiras de transformação política já tinham se tornado história, momento em que a própria esfera da política começou a experimentar um esvaziamento que só fez se acentuar desde então. [...] $\mathrm{O}$ reflexo, junto aos novos profissionais, do esmaecimento da política, e a hegemonia crescente do discurso técnico tornam a formação desses profissionais uma tarefa complexa, pois se de um lado é preciso dar-lhes uma formação teórica e técnica sólida, de outro é necessário que ela suscite uma vocação crítica e criativa, de modo a atender aos desafios que um processo de transformação contínuo, como a Reforma, impõe de modo constante. (BEZERRA JUNIOR, 2007, p. 245-246).

Também é crucial o confronto do conhecimento prévio do aluno com as novas realidades. Os Centros de atendimentos territoriais e não asilares deve servir de motivação para a compreensão de que os estigmas relacionados com a restrição social, incapacidades pessoais e distanciamento social não podem justificar a exclusão social.

De acordo com López et al. (2008) serviços de saúde mental tradicionais, como os hospitais psiquiátricos com base na lógica de exclusão social e separados do resto dos serviços de saúde, são um dos fatores que parece contribuir para o desenvolvimento e/ou reforço do estigma, o que fundamenta a discussão do uso dos dispositivos não asilares na educação, pois contribuem para reduzir estigmas muito associados à hospitalização, como os envoltos com a falta de capacidade de convício social e de independência, já que são serviços pautados na busca da autonomia do portador de transtorno mental.

Como em toda disciplina na área da saúde que trata de alguma patologia ou transtorno é fundamental que se estudem as condições determinantes que podem explicar o aparecimento de tal problema de saúde. Em se tratando de saúde mental, o modelo explicativo desses transtornos apresentados aos alunos pode estimular mesmo despropositalmente alguns estigmas.

Em relação aos modelos explicativos dos transtornos mentais, estes podem ser divididos, de acordo Bezerra Junior (2011), em reducionistas, que acreditam que toda experiência subjetiva tem explicação em bases biológicas, e em antirreducionistas, que acreditam que as bases biológicas são importantes, mas não suficientes para explicar as experiências subjetivas.

$\mathrm{O}$ modelo psicossocial de atenção à saúde Mental (antirreducionista) considera, de acordo com Luís (2011, p.55), que "fatores políticos, biopsíquicos e socioculturais sejam considerados determinantes das doenças". Portanto, como há a necessidade da interação de fatores diversos para o desenvolvimento de um determinado problema de saúde mental, a possibilidade é diminuída de os discentes apreenderem o transtorno mental como sendo fruto do individualismo. De acordo com o mesmo autor esse modelo "seria a estrutura perfeita para 
subsidiar a formação do profissional de saúde se não fosse a existência de contradições e conflitos gerados principalmente pelo fato de o modelo psicossocial não ser hegemônico" (LUIS, 2011, p. 56).

Um dos objetos de interesse do aluno durante a formação profissional são as categorias diagnósticas, que em algumas circunstâncias podem potencializar a estigmatização: "Pesquisas sugerem que muitas pessoas não procuram serviços de saúde mental porque não querem ser rotuladas como doentes mentais ou sofrer preconceito e discriminação que o rótulo implica" (CORRIGAN, 2007, p.31).

Essa forma de estigmatização, que para alguns pode ser sentida como ato de desconforto, descriminação ou marginalização, pode ser minimizada por meio da educação, que, segundo Pimentel; Villares; Mateus (2013) é o momento de estimular habilidades de comunicação que priorizam o uso cuidadoso da linguagem e das formas de abordar o portador de transtorno mental. Nesse processo educativo deve ser combatido o uso do diagnóstico como adjetivo pessoal, ou melhor, não confundir a pessoa com o seu diagnóstico.

Muito se fala dos estigmas associados a uma categoria diagnóstica que rotula o indivíduo, no entanto, algumas percepções estereotipadas acerca do portador de transtorno mental não estão associadas diretamente ao diagnóstico, mas com a forma ou ausência de tratamento e com a medicalização.

Apesar da diminuição progressiva da institucionalização dos portadores de transtornos mentais, essa lógica de tratamento é responsável pela manutenção atual de algumas deturpações da imagem do portador de transtorno mental, que ainda é caracterizado pela população como um indivíduo que apresenta características peculiares daqueles hospitalizados por longo tempo, o que Basaglia descreveu como uma síndrome encontrada em doentes mentais institucionalizados por longa data, caracterizando-se por:

\footnotetext{
Apatia, o desinteresse e o lento e monótono caminhar de cabeça baixa, sem rumo pelos corredores ou pelos pátios fechados, certos impulsos imotivados (com demasiada frequência reportados à doença); um comportamento submisso de animal domesticado, as lamúrias estereotipadas; o olhar perdido, desprovido de um ponto de apoio; a mente vazia porque não tem uma meta para qual voltar-se - estes são apenas alguns aspectos desta síndrome" (BASAGLIA, 2005, p. 50).
}

Essa imagem translocada que ocorre mesmo sem ocorrer internação advém em parte pela consolidação na sociedade dessa figura explicitada como síndrome da institucionalização, que ainda na atualidade acaba por se generalizar para todos os portadores de transtorno mental e é fortalecida pela mídia quando esta representa frequentemente o indivíduo como possuidor dessas características que funcionam como um estigma, já que não faz parte da realidade de seu transtorno e da vida de quem não está institucionalizado. 
O remodelamento do que é ensinado é possível desde que o programa educacional seja construído a favor do sujeito em sofrimento psíquico e não voltado simplesmente para a repetição de conhecimentos desatualizados e descontextualizados com o que é preconizado atualmente no campo da saúde mental, em especial no que concerne a prevenção e combate ao estigma.

\section{O contato como estratégia educacional voltada para o enfrentamento do estigma em saúde mental}

O contato com portadores de transtornos mentais por indivíduos em formação profissional é uma ferramenta que, quando apropriada pela educação, tem como fim a redução da estigmatização dos estudantes por meio da familiarização com indivíduos que apresentam algum sofrimento psíquico.

Esse tipo de estratégia antiestigma pode ser aplicado e analisado sob o ponto de vista retrospectivo, em que se observam as atitudes estigmatizantes de pessoas que já tiveram alguma experiência direta prévia com indivíduos em sofrimento psíquico, ou pelo enfoque prospectivo, onde o contato tem caráter experimental e que só posteriormente as mudanças atitudinais são avaliadas, o que López et al. (2008), consideram como mais favorável para melhor estabelecer os mecanismos intervenientes do contato.

Independente da sua natureza, o contato com os membros do grupo estigmatizado é considerado em vários como um meio efetivo para reduzir o preconceito intergrupal, considerando a estratégia mais eficaz para a melhoria de atitudes estigmatizastes. Nessa mesma direção, Pimentel, Villares e Mateus (2013), além de concordarem com essa funcionalidade do contato, também o consideram como a estratégia que mais reduz o estigma em longo prazo.

Para que as pessoas que possuam comportamentos estigmatizadores tenham atitudes modificadas elas devem ser familiarizadas a portadores de transtornos mentais que possam transmitir a sua experiência com o sofrimento psíquico, sua percepção do estigma e suas formas próprias de combatê-lo e desmistificá-lo, assim como o que espera de comportamento da sociedade perante a sua existência.

A estratégia de contato, ao permitir trocas entre teoria e prática traz benefícios para todas as partes integrantes da interação. Em relação ao portador de transtorno mental, desenvolve-se o sentimento de utilidade e capacidade em contribuir para a formação de alunos e profissionais, o que pode ser transformado em aumento da autoestima. Já para os alunos, "a oportunidade de ouvir sobre certos aspectos negativos que os usuários vivenciam, por vezes Barbarói, Santa Cruz do Sul, n. 56, p.<127-140>,jan./jun. 2020 
nos serviços, é uma experiência importante para que mudanças futuras sejam possíveis" (ELJAICK et al., 2016, p. 227).

O contato também se mostra útil quando favorece a integração dos participantes na vida cotidiana dos portadores de transtornos mentais, em que o contato se dá de forma naturalística, com oportunidades reais para interagir com esses indivíduos e longe dos padrões de um encontro controlado dentro de uma instituição, que pode não demonstrar a realidade das relações. De acordo com López et al. (2008), essa forma de interação oferece uma visão de pessoas que exercem funções normais, como, por exemplo, de um trabalhador, e que com isso se possa recategorizar e ajudar a reposicionar crenças e emoções por meio da interação de diferentes contextos.

Pelos resultados positivos encontrados em diversos estudos, a estratégia de contato deve ser considerada como uma aliada ao combate ao estigma. Todavia as intervenções antiestigmas podem ser potencializadas quando o contato está associado a outras abordagens educacionais. Conforme Oliveira, Carolino e Paiva (2012, p.35), "a utilização em conjunto do contato com a educação teórica gera mais opiniões favoráveis relacionadas ao transtorno mental, quando se compara com abordagens isoladas". Já Foster et al. (2010), diz que o contato direto ou indireto com indivíduos portadores de transtornos mentais aliado a educação em saúde, principalmente a voltada para o atendimento extra hospitalar, substitui mitos por realidade, afetando positivamente as atitudes em relação aos estigmas da periculosidade.

Os conhecimentos sobre as formas de melhor instituir o contato com portadores de transtornos mentais deve integrar o planejamento do ensino de Saúde Mental na educação profissional em saúde, pois é a partir dele que poderá se construir uma educação que permita avançar pelo difícil caminho da desestigmatização.

\section{Considerações finais}

A importância da educação profissional em consonância com teorias, práticas e atitudes, que possam restringir a ação do estigma associado ao transtorno mental, por parte dos profissionais da enfermagem, são essenciais. Justamente em um período em que se busca a consolidação de uma assistência humanizada e que tenta superar conceitos de saúde e doença que podem impulsionar estigmas e que ainda atravessam os programas de saúde mental.

Introduzir um ensino de saúde mental contextualizado com uma prática antiestigmatizante, na formação profissional dos trabalhadores da enfermagem produz inúmeras vantagens, como a melhora da assistência à saúde ao portador de transtorno mental, 
pois o bem estar provocado por um cuidado acolhedor, livre de estigmas provoca uma maior aderência ao tratamento proposto.

Para que esses propósitos possam ser alcançados, a formação profissional deve priorizar uma educação voltada para geração de profissionais da enfermagem competentes a contextualizar suas práticas com a realidade social. Assim, consegue-se formar muito mais que trabalhadores da saúde, mas sujeitos políticos com capacidade transformadora no campo da atenção psicossocial.

O profissional da enfermagem quando tem seus saberes e práticas reformulados percebe com mais clareza que sua função, além do cuidado direto ao indivíduo com algum sofrimento psíquico, é de ser mais um combatente do estigma.

\section{CONTRIBUCIONES DE LA EDUCACIÓN PROFESIONAL EN LA ENFERMERÍA PARA EL ENFRENTAMIENTO DE LA ESTIGMATIZACIÓN ASOCIADA A LOS TRASTORNOS MENTALES}

\section{Resumen}

As actitudes estigmatizadas asociadas con los pacientes con trastornos mentales presentes en los estudiantes y los trabajadores de la salud reproducir en el cuidado de la salud, produciendo frustración, además del trastorno mental en sí. Una forma de enfrentar este problema social es invertir en educación profesional en educación antiestigmatizante. Para contemplar un tema, se realizó una revisión de la literatura para lograr los siguientes objetivos de investigación, que son diseñar señalandos teóricos y prácticos sobre educación, durante una capacitación profesional, podría ser una forma de enfrentar el estigma relacionado con las personas con las personas trastornos mentales, presente en el área de enfermería. Un argumento central del artículo es que la capacitación profesional es un momento importante para combatir la estigmatización de las personas con trastornos mentales. Por lo tanto, es esencial que la educación en salud mental se base en la educación crítica, tenga sus contenidos formados en un contexto antiestigmatizante y asocie varias estrategias educativas destinadas a reducir el estigma, como ocurre en la estrategia de contacto con personas con trastornos mentales. La educación profesional de enfermería, en línea con una práctica antiestigmatizante, puede restringir una acción de estigma asociada con el trastorno mental por parte de los profesionales de enfermería. La consecuencia de este tipo de capacitación es la mejora de la atención médica debido a un menor estigma médico.

Descriptores: Estigma social; Transtornos mentales; Educación; Enfermería

\section{CONTRIBUTIONS OF PROFESSIONAL EDUCATION IN NURSING FOR THE FIGHTING OF STIGMATIZATION ASSOCIATED WITH MENTAL DISORDERS}

\section{Abstract}

The stigmatized attitudes associated with patients with mental disorders present in students and health workers are reproduced in health care, producing suffering, in addition to caused by the mental disorder itself. To contemplate the theme, a literature review was carried out so that the following research objectives achieved, which is to conceive theoretical and practical notes on how education, during professional training, can be a way of dealing with the stigma 
related to patients mental disorders, present in the nursing area. A central argument of the article is that professional training is an important moment to combat the stigmatization of people with mental disorders. Therefore, it is essential that mental health education be based on critical education, have its formative content in an anti-stigmatizing context and associate several educational techniques aimed at reducing stigma, as occurs in the strategy of contact with people with mental disorders. Nursing professional education when in line with an antistigmatizing practice is able to restrict an action of stigma associated with mental disorder, by nursing professionals. The consequence of this type of training is the improvement of assistance due to health care with less stigma.

Keywords: Social stigma; Mental disorders; Education; Nursing

\section{REFERENCIAS}

AMARANTE, Paulo (Org.). Cultura da formação: reflexões para a inovação no campo da saúde mental. In: AMARANTE, Paulo; CRUZ, Leandra Brasil da (Org.). Saúde Mental, Formação e Crítica. Rio de Janeiro: Laps, 2008. p. 65-78.

AHMEDANI, Brian K. Mental Health Stigma: Society, Individuals, and the Profession. J Soc Work Values Ethics, v. 2, n. 8, p.1-16, 2011. Disponível em:

<https://www.ncbi.nlm.nih.gov/pmc/articles/PMC3248273/>. Acesso em: 05 nov. 2016.

ARBOLEDA-FLÓREZ, Julio; STUART, Heather. From Sin to Science: Fighting the Stigmatization of Mental Illnesses. Revue Canadienne de Psychiatrie, Canadá, v. 57, n. 8, p.457-463, 2012. Disponível em: <http://connection.ebscohost.com

/c/articles/78854969/from-sin-science-fighting-stigmatization-mental-illnesses $>$. Acesso em: 09 ago. 2016.

\section{BASAGLIA, Franco. Escritos selecionados em saúde mental e Reforma}

Psiquiátrica.(Org.) Paulo Amarante. Rio de Janeiro: Garamond, 2005.

BATISTA, Luísa Teixeira Martins Barreira. Os profissionais de saúde e o estigma da doença mental. 2013. 32 f. Dissertação (Mestrado) - Curso de Medicina, Universidade do Porto, Porto, 2013. Disponível sigarra.up.pt/ffup/pt/pub_geral.show_file?pi_gdoc_id=613265>. Acesso em: 05 nov. 2016.

BEZERRA JUNIOR, Benilton. Desafios da Reforma Psiquiátrica no Brasil. Physis: Rev. Saúde Coletiva, Rio de Janeiro, v. 2, n. 17, p.243-250, 2007. Disponível em:

<http://www.scielo.br/scielo.php? script=sci_arttext\&pid=S0103-73312007000200002>. Acesso em: 05 nov. 2016.

BEZERRA JUNIOR, Benilton. Há fatos que resistem a explicações simples. Poli, Rio de Janeiro, p.21-23, mai/jun. 2011.

CORRIGAN, Patrick W. How Clinical Diagnosis Might Exacerbate the Stigma of Mental Illness. Soc Work, Si, v. 1, n. 52, p.31-39, jan. 2007.

EL-JAICK, F.S., et al. Usuários da saúde mental como educadores: o que suas narrativas podem nos ensinar?. Interface - Comunicação, Saúde, Educação, Botucatu, v. 20, n. 56, p.227-238, mar. 2016. 
FOSTER, K., et al. Mental health worker's attitudes toward mental illness in Fiji. Australian Journal of Advanced Nursing, South Melbourne, v. 25, n. 3, p. 72-9, 2010.

GIL, Isabel Maria de Assunção. Crenças e Atitudes dos Estudantes de Enfermagem acerca das Doenças e Doentes Mentais: Impacto do Ensino Clínico de Enfermagem de Saúde Mental e Psiquiatria. 2010. 115 f. Dissertação (Mestrado) - Curso de Medicina, Universidade de Coimbra, Coimbra, 2010. Disponível em:

<https://estudogeral.sib.uc.pt/handle/10316/18217>. Acesso em: 05 nov. 2016.

GYLLENSTEN, Amanda Lundvik et al. Attitudes in Healthcare Students Towards Mental Illness a pre and post Multicenter University Program Survey. Ijahsp, Chicago, v. 9, n. 3, p.18, 2011. Disponível em: <http://nsuworks.nova.edu/ijahsp/vol9/iss3/11/>. Acesso em: 04 mar. 2017.

LUCCHESE, Roselma; BARROS, Sônia. A constituição de competências na formação e na prática do enfermeiro em saúde mental. Revista da Escola de Enfermagem da Usp, [s.1.], v. 43, n. 1, p.152-160, mar. 2009.

LÓPEZ, Marcelino et al. La lucha contra el estigma y la discriminación en salud mental: Una estrategia compleja basada en la información disponible. Revista de La Asociación

Española de Neuropsiquiatría, [s.1.], v. 28, n. 1, p.43-83, 2008.

LUIS, Margarita Antônia Villar. Os rumos do cuidado em Saúde Mental. In: SOARES, Marcos Hirata; BUENO, Sonia Maria Villela (Org.). Saúde Mental: Novas Perspectivas. São Paulo: Yendis, 2011. p. 55-68.

OLIVEIRA, Sandra; CAROLINO, Luísa; PAIVA, Adriana. Programa Saúde Mental Sem Estigma: Efeitos de Estratégias Diretas e Indiretas nas Atitudes Estigmatizantes. Revista Portuguesa de Enfermagem de Saúde Mental, Porto, n. 8, p.30-37, 2012. Disponível em: $<$ http://www.scielo.mec.pt/scielo.php?script=sci_arttext\&pid=S1647-21602012000200005>. Acesso em: 05 nov. 2016.

PIMENTEL, Fernanda de Almeida; VILLARES, Cecília Cruz; MATEUS, Mário Dinis. Estratégias de combate ao estigma na saúde mental. In: MATEUS, Mário Dinis (Org.). Políticas de saúde mental: Baseado no curso Políticas públicas de saúde mental, do CAPS Professor Luiz da Rocha Cerqueira. São Paulo: Instituto de Saúde, 2013. p. 306-321. Polit DF, Hungler BP. Fundamentals of nursing research. 4a ed. Porto Alegre: Artes Médicas; 2004.

THORNICROFT, Graham et al. Reducing stigma and discrimination: candidate interventions. International Journal Of Mental Health Systems, [s.1.], v. 2, n. 1, p.1-7, abr.2008.

THORNICROFT, Graham et al. Stigma: ignorance, prejudice or discrimination? British Journal Of Psychiatry, [si], v. 190, p.192-193, 2007. Disponível em:

http://bjp.rcpsych.org/>. Acesso em: 19 ago. 2016.

Data de recebimento: 29/04/2019

Data de aceite: 01/06/2020 


\section{Sobre as autoras:}

Marcela Santos Ferreira é Enfermeira, Mestre em Educação Profissional em Saúde pela Escola Politécnica em Saúde Joaquim Venâncio/FIOCRUZ. Docente do curso de Enfermagem do Centro Federal de Educação Tecnológica Celso Suckow da Fonseca/ CEFETRJ. Endereço Eletrônico: cceccella@ hotmail.com

Maria Cecilia Araújo Carvalho é Médica, doutora em Psiquiatria, Psicanálise e Saúde Mental pela Universidade Federal do Rio de Janeiro. Pesquisadora e docente da Escola Politécnica de Saúde Joaquim Venâncio da Fundação Oswaldo Cruz. Endereço Eletrônico: carvalho.mariacecilia@gmail.com 